\title{
METHOD BASED ON IMPROVED FASTICA-LSM FOR Harmonic Detection In Power System
}

\author{
Xiaojuan Chen ${ }^{1}$, Jie $\mathrm{Wu}^{2}$, Xinxin $\mathrm{Fan}^{3}$, Meng $\mathrm{Xu}^{3}$ and Yinghui Zhao ${ }^{3}$ \\ ${ }^{1}$ Institute of Electronic Information Engineering, Changchun University of Science and \\ Technology, Chang Chun, China \\ 919149563@qq.com \\ ${ }^{2}$ ElectricalEngineering, Beihua University, Jilin, China \\ Fanxxok@126.com \\ ${ }^{3}$ Institute of Information Engineer, Northeast Dianli University, Jilin, China \\ xumeng9309@163.com
}

\begin{abstract}
This paper proposes a new method based on improved FastICA (fast independent component analysis)LSM (the least square method), in order to improve the accuracy and speed of harmonic detection in power system. This work ameliorates the traditional FastICA algorithm by defining the initial value of demixing matrix iteration, thus inducing the iteration times and eliminating the problem of higher-order convergence FastICA sensitive to initial value, and harmonics and fundamental components are extracted from electric signals using fifth-order convergence FastICA. Finally LSM is used to calculate the amplitude and phase angle of separation components. Simulated and real signals are used to test the method. Results shows that the proposed method has faster detection rate and higher accuracy compared with other methods available in the literature.
\end{abstract}

\section{KEYWORDS}

Harmonic detection, FastICA, Initial value definition, fifth-order convergence, LSM

\section{INTRODUCTION}

Increasing use of nonlinear equipments cause a mass of harmonic in power system recently, which is serious both for consumers and the efficiency of power supply and distribution in power grid.

Many harmonic analysis methods have been developed presently, such as FFT (Fast Fourier Transform), instantaneous reactive power, WT (wavelets transform), ICA (independent component analysis) and neural network, etc[1,2]. Among them, method based on FFT is the most widely used method because of its relatively stable performance, however spectrum leakage and fence effect would exist to cause more error[3]. Method based on WT could overcome this deficiency, however it is susceptible to the choice of wavelet basis[4]. Harmonic detection based on instantaneous reactive power needs lots of hardware [5].

ICA have advantages of simple calculations, stable performance comparing with them, what's more, it can extract components with few prior knowledge. Literature [6] applied ICA in harmonic detection and using both simulated and real signals to test the method. In literature [7, 8] FastICA with third-order convergence was proposed to improve the speed of harmonic detection, but it couldn't estimate amplitudes and phases of harmonics accurately. Method based 
on FastICA combining with LSM (the least squares method) is proposed in Literature [9], the LSM was used to determine amplitudes and phases of harmonic, however the main disadvantage is that the method can't meet a good real-time operation.

This paper uses fifth-order convergent FastICA to extract harmonic and fundamental components from electric signals and iteration's initial value of des-mixing matrix is defined when calculating it. Finally LSM is used to calculate the amplitude and phase angle of separation components.

\section{INDEPENDENT COMPONENT ANALYSIS}

\subsection{FUNDAMENTALS OF INDEPENDENT COMPONENT ANALYSIS}

ICA is an algorithm developed on the basis of BBS (Blind Source Separation). The original components to be separated are considered statistically independent and follow a non-Gaussian distribution. It stated as a set of input independent signals $S=\left[s_{1}, s_{2}, s_{3} \ldots s_{m}\right]^{T}$ which represents orginal signal in this work, where $m$ denotes time or sample sequence. Thus observed matrix $\boldsymbol{X}$ $=\left[x_{1}, x_{2}, x_{3} \ldots x_{n}\right]^{T}, n \geq m$ is got, which denote electric signals in this work, through a $n \times m$ dimension mixture matrix $\boldsymbol{A}$. Relationship of observed matrix and source can use:

$$
X=A S
$$

The purpose of ICA is that when the source and mixture matrix are unknown, des-mixing matrix $\mathrm{W}$, an estimate of A-1 by optimization algorithm, is obtained using only the observed signals. Thus S's optimum estimate are obtained by

$$
Y=W X=W A S
$$

FastICA, a fast optimization iteration algorithm based on fixed-point recursive, is a kind of ICA often used. It has pretreatment of observation and independent components extraction, and there are two parts in observed pretreatment: signal centralized and whiten.

Obtaining the separation matrix $\mathrm{W}$ by iterative algorithm is an indispensable part of independent component extraction, and maximum negentropy is adopted as criterion to measure non-Gaussian of separated components in this context. For y is a random variable, it's probability density is $\mathrm{p}(\mathrm{y})$, the negative entropy approximate expression can be described:

$$
J(y) \propto\left[E\{G(y)\}-E\left\{G\left(y_{y=s u s}\right)\right\}\right]^{2}
$$

Where y is a Gaussian random vector which has the same covariance with y, $\mathrm{G}(\cdot)$ is a function both non-quadratic and non-linear, $\mathrm{E}(\cdot)$ is algorithm that calculate mean $[10,12]$.

Central-limit theorem shows that y is non-Gaussian distribution when formula (3) is maximum, and the maximum of negative entropy can be acquired by Newton iteration method which is quadratic convergent, thus getting iteration formula simplified of demixing

$$
w^{a}=E\left\{x g\left(w^{T} x\right)\right\}-E^{\prime}\left\{g^{\prime}\left(w^{T} x\right)\right\} w
$$

This result is normalized by

$$
w^{*}=w^{a} /\left\|w^{a}\right\|
$$

When the result of formula (4) is convergent, $w^{a}$ is the needed elements of $\boldsymbol{W}$, thus demixing matrix $\mathrm{W}$ is obtained $[13,14]$. 


\subsection{IMPROVED FASTICA}

The initial value of iteration is selected randomly when solving demixing matrix in the traditional FastICA, which is two order convergence and can't meet the highly demanded real-time for harmonic detection in power system. Amendatory iterative formula which is fifth-order convergent is used here, however FastICA with higher-order convergence is sensitive to initial. Non-convergent will be generated, if improper initial value is chosen. This work, initial value of des-mixing matrix iteration is defined when calculating it, through hypothesizing the original source signals' components according to the characteristics of power system harmonics, thus inducing the iteration times and eliminating the problem of higher-order convergence FastICA sensitive to initial value.

\subsubsection{FASTICA With FifTh-ORDER CONVERGENCE}

Newton iteration with quadratic convergence corrected to fifth-order convergent can be expressed as follows:

$$
x_{n+1}^{*}=x_{n}-\frac{f\left(x_{n}\right)}{f^{\prime}\left(x_{n}\right)} ; u_{n+1}=x_{n}-\frac{f\left(x_{n}\right)}{2}\left(\frac{1}{f^{\prime}\left(x_{n}\right)}+\frac{1}{f^{\prime}\left(x_{n+1}^{*}\right)}\right) ; x_{n+1}=u_{n+1}-\frac{f\left(u_{n+1}\right)}{f^{\prime}\left(\frac{1}{2}\left(x_{n+1}^{*}+u_{n+1}\right)\right)}, n=1,2 \Lambda
$$

It can be proved that formula (6) is fifth-order convergent. Then new FastICA formula with relaxation factor obtained by formula (6) is shown as following:

$$
\begin{aligned}
& w_{i}^{\#}=E\left[x g\left(w_{i}^{T} x\right)\right]-E\left[g^{\prime}\left(w_{i}^{T} x\right)\right] \cdot w_{i}-\beta\left(1-\alpha_{i}\right) w ; w_{i}^{*}=2 E\left[x g\left(w_{i}^{T} x\right)\right]-\left\{E\left[g^{\prime}\left(w_{i}^{\# T} x\right)\right]+E\left[g^{\prime}\left(w_{i}^{T} x\right)\right]\right\} \cdot w_{i} \\
& w_{i}=E\left[x g\left(w_{i}^{* T} x\right)\right]-E\left\{\frac{1}{2}\left[\left(g^{\prime}\left(w_{i}^{* T} x\right)+g^{\prime}\left(w_{i}^{\# T} x\right)\right]\right\} \cdot w_{i}^{*} ; \quad w_{i}=\frac{w_{i}}{\left\|w_{i}\right\|}\right.
\end{aligned}
$$

where $\beta=E\left\{w_{i}^{T} x g\left(w_{i}^{T} x\right)\right\}, \alpha_{i}$ is relaxation factor ${ }^{[15]} \cdot w_{i}$ is the needed element of $\boldsymbol{W}$ when formula (7) is convergent.

\subsubsection{DeFinition OF The INITIAL VALUE}

Electric signal containing harmonics is modeled as[16]:

$$
s(t)=s_{f}(t)+s_{d}(t)=\sum_{h} U_{h} \sin \left(h \omega t+\theta_{h}\right), \quad h=1, \quad 3, \quad 5 \Lambda
$$

It is assume that $s(t)$ is voltage signals, where $U_{h}$ and $\theta_{h}$ are amplitude and phase angle of the $h$ th harmonic, $\omega$ is the fundamental frequency in rad/s, $s(t)$ is fundamental voltage when $h$ equaling 1 . The number of harmonic signal can be determined in consideration of noise by the following way: First, autocorrelation function is estimated according to electric signals, then here construct autocorrelation matrix and the Eigen values of matrix is decomposed to determine the number of harmonic signal[17] Since low-order harmonics result more damage to power grid, it assume that harmonics included in source signals obey the principle that from low order to high order. Such as a observed signal is determined containing 4 harmonic components, thus it can assume that the electric signal $s(t)$ consists of fundamental signal and 3, 5, 7, 9 harmonics as source signal with zero phases, that is to say $h$ is 9 here. Then source matrix $S$ obtained from $s(t)$ can be represented by: 
Electrical and Electronics Engineering: An International Journal (ELELIJ) Vol.5 , No.4, November 2016

$$
S=\left[\begin{array}{c}
U \sin (\omega t) \\
U_{3} \sin (3 \omega t) \\
U_{5} \sin (5 \omega t) \\
U_{7} \sin (7 \omega t) \\
U_{9} \sin (9 \omega t)
\end{array}\right]
$$

Then demixing matrix $\boldsymbol{W}$ can be calculated by formula (2) when observed signal is constructed and it is plugged into iterative formula as iterative value.

\section{METHOD BASED ON IMPROVED FASTICA-LSM FOR HARMONIC DETECTION}

\subsection{ObSERVATion Signals CONSTRUCTION}

Electric signal in power system as the observed signal is single channel, while fundamental signal and the harmonics as source are multi-channel, so it is necessary to form a multi-channel observed signal whose number is equal to the source signal. This work can be done by generating vectors with time delays from observed signal $x(t)$ [18], observation matrix can use

$$
X(t)=\left[\begin{array}{c}
x(t) \\
x(t-1) \\
\mathrm{M} \\
x(t-k)
\end{array}\right] .
$$

\subsection{HARMONIC COMPONENTS EXTRACTED BASED ON IMPROVED FASTICA}

Steps of Harmonic components extraction based on improved FastICA as following:

Step 1 Centralize the observed signals;

Step 2 White centralized signals;

Step 3 Initializes the demixing matrix;

Step 4 Calculate initialization of demixing by the method presented at subsection 2.2.2.

Step 5 Obtain demixing matrix use formula (7);

Step 6 Orthogonalize the demixing matrix got from Step 5;

Step 7 Determine whether the row vector obtained by step 5 if convergence or not, if not, repeat Step 3;

Step 8 Recover source signals from separated components obtained by demixing matrix.

\subsection{Amplitude And Phase Of The Signal Calculation Based On LSM}

FastICA can't ensure the amplitude and phase of signal exactly, LSM is used here to calculate them like literature [9] expressed. Formula(8) can be decomposed as

$$
x(t)=\sum_{h} U_{h} \sin \left(h \omega t+\theta_{h}\right)=\sum_{h} A_{h} \sin (h \omega t)+B_{h} \cos \left(h \omega_{f} t\right)
$$

where $A_{h}=U_{h} \cos \theta_{h}, B_{h}=U_{h} \sin \theta_{h}$. Formula (11) can be expressed using another formula

$$
X=H I+e
$$

where $\boldsymbol{X}$ is observed signal, 
Electrical and Electronics Engineering: An International Journal (ELELIJ) Vol.5 , No.4, November 2016

$$
H=\left[\begin{array}{cccc}
\sin \left(\omega_{1} t_{1}\right) & \cos \left(\omega_{1} t_{1}\right) & \Lambda & \cos \left(N \omega_{N} t_{1}\right) \\
\sin \left(\omega_{1} t_{2}\right) & \cos \left(\omega_{1} t_{2}\right) & \Lambda & \cos \left(N \omega_{N} t_{2}\right) \\
\mathrm{M} & \mathrm{M} & & \mathrm{M} \\
\sin \left(\omega_{1} t_{N}\right) & \cos \left(\omega_{1} t_{N}\right) & \Lambda & \cos \left(N \omega_{N} t_{N}\right)
\end{array}\right]
$$

$I=\left[\begin{array}{lllll}A_{1} & B_{1} & \Lambda & A_{N} & B_{N}\end{array}\right]$, e is error vector. LSM is used to pre-estimate I by

$$
I=\left(H^{\mathrm{T}} H\right)^{-1} H^{T} X
$$

where $\boldsymbol{H}$ is known from separated source component by FastICA, and then get coefficient matrix $\boldsymbol{I}$, thus amplitude and phase of the signals can be calculated by:

$$
\left\{\begin{array}{l}
U_{h}=\sqrt{A_{h}^{2}+B_{h}^{2}} \\
\theta_{h}=\arctan \frac{A_{h}}{B_{h}}
\end{array}\right.
$$

\section{EXPERIMENTAL RESULTS}

\section{Case 1}

A source signal consisting of fundamental signal and third, fifth, seventh harmonics was constructed and signal-to-noise ratio (SNR) of $20 \mathrm{~dB}$ was considered according to the characteristic of harmonics in power system, which is modeled as:

$$
x(t)=\sin (100 \pi t)+0.35 \sin \left(300 \pi t+\frac{\pi}{7}\right)+0.18 \sin \left(500 \pi t+\frac{\pi}{5}\right)+0.12 \sin \left(100 \pi t+\frac{\pi}{6}\right)
$$

Fig. 1 and Fig. 2 show the source signals and mixed signal obtained from original source.

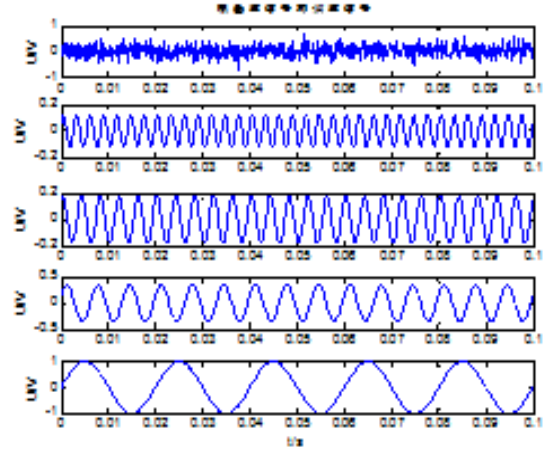

Fig.1 Source signals

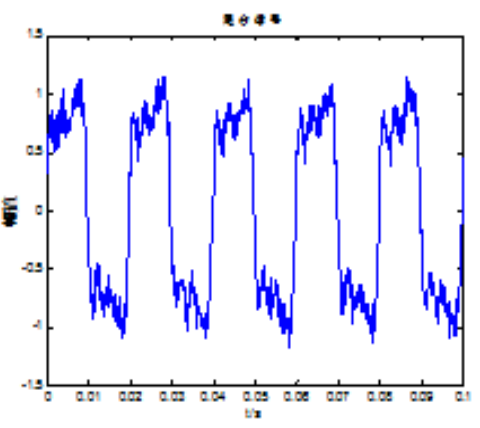

Fig.2 Mixed signal

The improved FastICA, fifth-order convergence FastICA but no defining initial value and traditional FastICA are defined as method 1, method 2 and method 3 to process the obverted signal. Results of separated components obtained by these three methods are shown in Fig. 3. 


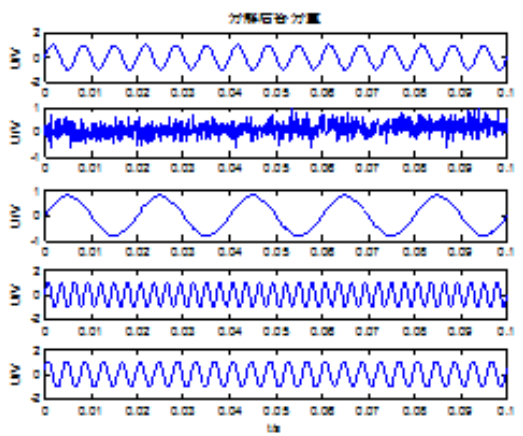

(a) Separated signals by method 1

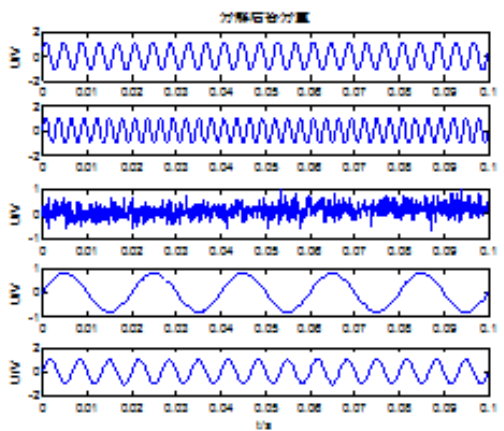

(b) Separation signals by method 2

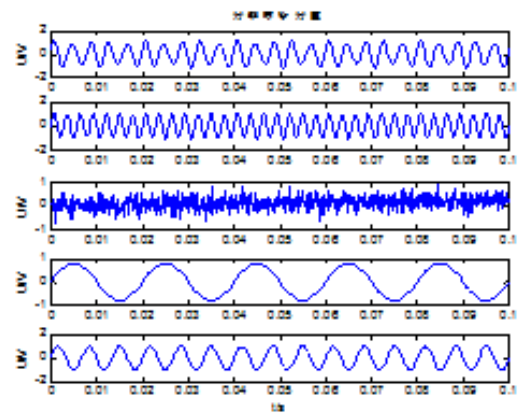

(c) Separation signals by method 3

Fig.3 Separation signals by three methods for case 1

The results can be seen that separated signals based on method 1 and 2 are closer to the orginal source signals. But it wouldn't convergent sometimes and lead to test failure when method 2 was used.

LSM was used to calculate amplitude and phase angle of separated components, which is got based on method 1, 2, 3 for case 1 and using the same one platform to test through the three methods for 1200 sample points. results are shown in Table. 1,

Table.1: Separetion results and run-time of the three methods

\begin{tabular}{|c|c|c|c|c|c|}
\hline Meth & Extracted & Amplitude(V) & Amplitude's & Phase angle's Mean & Run-time(s) \\
\hline \multirow{4}{*}{1} & Base wave & 0.9901 & \multirow{4}{*}{$2.6 \%$} & \multirow{4}{*}{$3.8 \%$} & \multirow{4}{*}{0.462} \\
\hline & 3rd harmonic & 0.3583 & & & \\
\hline & 5th harmonic & 0.1729 & & & \\
\hline & 7th harmonic & 0.1135 & & & \\
\hline \multirow{4}{*}{2} & Base wave & 0.9876 & \multirow{4}{*}{$4.1 \%$} & \multirow{4}{*}{$5.1 \%$} & \multirow{4}{*}{0.478} \\
\hline & 3rd harmonic & 0.3407 & & & \\
\hline & 5th harmonic & 0.1739 & & & \\
\hline & 7th harmonic & 0.1308 & & & \\
\hline \multirow{4}{*}{3} & Base wave & 0.8876 & \multirow{4}{*}{$11.3 \%$} & \multirow{4}{*}{$52.2 \%$} & \multirow{4}{*}{0.909} \\
\hline & 3rd harmonic & 0.3217 & & & \\
\hline & 5th harmonic & 0.1979 & & & \\
\hline & 7th harmonic & 0.1008 & & & \\
\hline
\end{tabular}


Tab. 1 shows that the detection accuracy based on method 1 and 2 are better than method 3 .

\section{Case 2}

To prove practicability of method, A real electric signal taken from the IEEE working group P1159.3 dataset[18] were presented to test. The signal represent the current of a transformer at some moment and the waveform of it is shown as Fig.4, Results of separated signals obtained based on method 1 and method 3 are shown in Fig. 5, Fig. 6, and when method 2 was used it wouldn't convergent sometimes and lead to test failure.

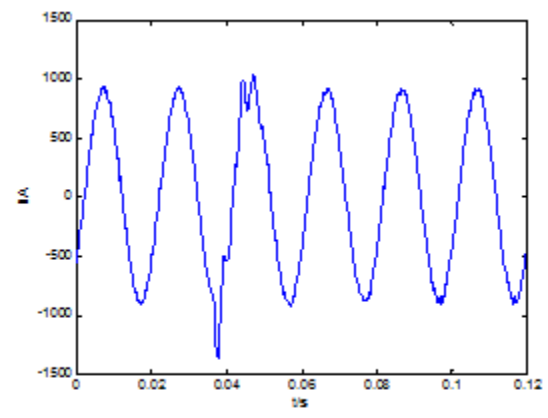

Fig.4 Current signal

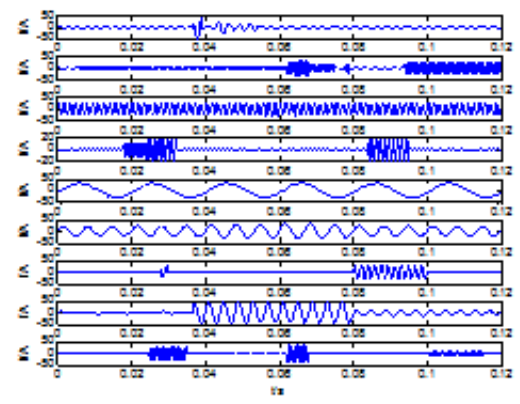

Fig. 5 Separation components by method 1

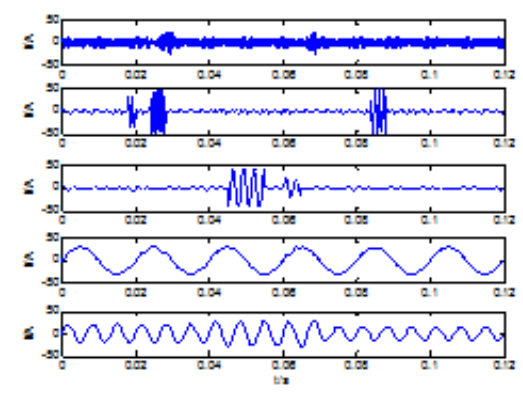

Fig.6 Separation components by method 3

The results can be seen that nine separated signals were extracted from electric signal based on the proposed method here, while five separated signals were extracted based on traditional FastICA, and in the view of the fundamental waveform obtained by the two methods, the former has litter distortion. Since harmonics extracted from electric are a periodic, it is difficult and needless to use LSM calculating their amplitude and phase. The results of amplitude and phase of fundamental signal obtained from electric signal based on the two methods and run-time are shown in Table 2.

Table.2 Amplitude and phase of fundamental signal and run-time

\begin{tabular}{|c|c|c|c|}
\hline & Amplitude(A) & Phase $\left({ }^{\circ}\right)$ & Run-time(s) \\
\hline Improved FastICA & 901.348 & 24.861 & 0.289 \\
\hline FastICA & 880.653 & 20.092 & 0.931 \\
\hline
\end{tabular}

Table. 2 shows that the time used based on method 1 is less by $69.5 \%$ than method 3, which proving the efficiency of the proposed method based on improved FastICA-LSM. 


\section{CONCLUSION}

This paper combine FastICA with LSM detect harmonics in power system, mitigating the problems of speed and accuracy are contradictory in traditional power system harmonic detection methods. Traditional FastICA initial value is chosen at random, so it have more iterations, and higher order convergence algorithm are sensitive to initial. According to this problem, this work The method uses fifth-order convergent FastICA algorithms to extract harmonic signals and define fundamental signal from electrical signals.

\section{ACKNOWLEDGEMENTS}

This work is partially supported by the National Nature Science Foundation of China (NNSFC) (Grant No. 61271115) for which we are extremely grateful. The authors also gratefully acknowledge the helpful comments and suggestions of the reviewers, which have improved the presentation.

\section{REFERENCES}

[1] Xiong J F, Li Q. \& Yuan X D, (2013) "Detection Methods of Harmonics and Inter-harmonics in Power Systems", Automation of Electric Power Systems, Vol. 37, No. 11, pp125-133.

[2] Duque C A, Silveira P M. \& Ribeiro P F, (2008) "Visualizing time-varying harmonics using filter banks", Electric Power Systems Research, Vol. 81, No. 4, pp947-983.

[3] Fang G Z, Yang C. \& Zhao H, (2012) "Detection of harmonic in power system based on FFT and wavelet packet ", Power System Protection and Control, Vol. 40, No. 5, pp75-79.

[4] Zhou L H, Fu Q. \& Yu S J. Harmonic detection based on wavelet transform", Proceedings of the CSU-EPSA, Vol. 22, No. 1, pp80-85.

[5] Nath S, Sinha P. \& Goswami S K, (2012) "A wavelet based novel method for the detection of harmonic sources in power systems", International Journal of Electrical Power \& Energy Systems, Vol. 40, No. 1, pp54-61.

[6] Ferreira D D, Nagata E A. \& Ferreira S C, (2015) " Method based on independent component analysis for harmonic extraction from power system signals", Electric Power Systems Research, Vol. 37, No. $11, \mathrm{pp} 119-124$.

[7] Wang J, Wang N. \& Wang L, (2010) " Harmonic detection of power quality based on improved FastICA", Power System Protection \& Control, Vol. 38, No. 18, pp126-130.

[8] Wang B, Wang N. \& Jiang Y, (2011) “Application of improved FastICA in harmonic detection", Electric Power Automation Equipment, Vol. 31, No. 3, pp135-138.

[9] Meng L L, Sun C D. \& Han B R, (2012) "Algorithm for inter-harmonic detection based on least square method and ICA ", Power System Protection and Control, Vol. 40, No. 11, pp76-81.

[10] Gursoy E. \& Niebur D, (2009) "Harmonic Load Identification Using Complex Independent Component Analysis", IEEE Transactions on Power Delivery, Vol. 24, No. 1, pp285-292.

[11] Gouy-Pailler C, Congedo M. \& Brunner C, (2010) " Nonstationary Brain Source Separation for Multiclass Motor Imagery”, Biomedical Engineering IEEE Transactions on, Vol. 57, No. 2, pp469478.

[12] Reyhani N, Ylipaavalniemi J. \& Ricardo Vigário, (2012) "Consistency and asymptotic normality of FastICA and bootstrap FastICA", Signal Processing, Vol. 92, No. 8, pp1767-1778.

[13] Khoury G E, Nechepurenko Y M. \& Sadkane M, (2014) "Acceleration of inverse subspace iteration with Newton's method", Journal of Computational \& Applied Mathematics, 259: 205-215. Vol. 92, No. 8, pp205-215.

[14] Xu L, (2015) "Application of the Newton iteration algorithm to the parameter estimation for dynamical systems", Journal of Computational \& Applied Mathematics, Vol.26, No. 8, pp33-43.

[15]Meng L, Sun C. \& Wang X, (2012) "A Detection Method for Harmonic/Inter-harmonic Based on Eigenvalue Decomposition and Fast Independent Component Analysis", Automation of Electric Power Systems, Vol. 36, No. 5, pp61-66.

[16] Xiao Z, Gong R. \& Chen S, (2015) “ Detection of harmonics in power system based on modified Stransform”, Power System Protection \& Control, Vol. 43, No. 3, pp84-90. 
Electrical and Electronics Engineering: An International Journal (ELELIJ) Vol.5 , No.4, November 2016

[17] Yin H W, Li G L. \& Lu G H, (2014) “An Improved Double Factor Adaptive Fast ICA Algorithm”, Journal of Sichuan University, Vol. 46, No. 6, pp128-132.

[18] Zheng D F, Chen H K. \& Yang Z P, (2012) “Application of Independent Component Analysis on Harmonic Source Identification”, Proceedings of the Chinese Society of Universities for Electric Power System \& Its Automation, Vol. 24, No. 4, pp134-144.

\section{AUTHORS}

Xiaojuan Chen was born in Changchun, Jilin Province, in 1970. He received the B.S degree from Institute of Information Engineer, Northeast Dianli University and the $\mathrm{Ph}$. D. degree from Jilin University in 2005 and 2009, respectively. He is now a professor and also supervisor of postgraduate. His research concerns Power device noise testing and Reliability analysis of power carrier.

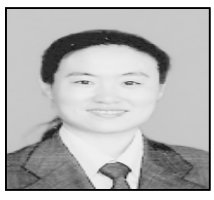

Xinxin Fan was born in Bozhou, Anhui Province, in 1990.. He is now an enroll ed postgraduate at Northeast Dianli University. His research concerns the detection of the low frequency noise of electronic device.

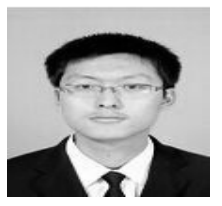

